УДК 551.590 .2

\title{
ПЕРИОДИЧЕСКИЕ ИЗМЕНЕНИЯ ПРИЗЕМНОЙ ТЕМПЕРАТУРЫ ВОЗДУХА, ВЫЗВАННЫЕ ВЛИЯНИЕМ КОСМИЧЕСКИХ ФАКТОРОВ
}

\author{
М.Б. Богданов, А.В. Катрущенко \\ Саратовский государственный университет, \\ кафедра метеорологии и климатологии \\ E-mail: BogdanovMB@info.sgu.ru
}

Проведен анализ спектра мощности временного ряда среднесуточных значений приземной температуры воздуха в г.Саратове на интервале времени с 1945 по 1999 г. В спектре ряда обнаружены две приливные гармоники, гармоника с периодом синодического месяца, связанная с изменением инсоляции при движении системы Земля - Луна вокруг общего центра масс, а также гармоника, соответствующая среднему периоду обращения активных областей Солнца. Проявлений 11-летнего цикла солнечной активности и периодических изменений инсоляции, вызванных планетными возмущениями орбиты Земли, не обнаружено.

Ключевые слова: воздух, приземная температура, г. Саратов, гармоники, космические факторы.

Periodical Changes in the Surface air Temperature, Caused by the Influence of the Space Factors

\section{M.B. Bogdanov, A.V. Katrushchenko}

We carried out the analysis of the power spectrum of the time series of the daily mean values of the surface air temperature in Saratov city (Russia) in the time interval from 1945 through 1999. In the power spectrum are discovered two tidal harmonics, the harmonic with the period of synodic month, connected with a change in the insolation during the motion of system the Earth - Moon around the common center of masses, and also harmonic, which corresponds to the average period of rotation of the active regions of the Sun. The manifestations of the 11-year solar cycle and changes in the insolation, caused by the planetary perturbations of the Earth's orbit, are not discovered.

Key words: ground air temperature, Saratov, harmonic components, space factors.

\section{Введение}

В последние годы многими представителями наук о Земле исследуется проблема влияния космических факторов на процессы, протекающие в глубинах Земли, ее атмосфере и гидросфере. В число этих факторов входят разнообразные проявления солнечной активности [1], особенности движения Земли в Солнечной системе, ее вращения вокруг собственной оси и движения полюса $[2,3]$, а также приливные воздействия Луны и Солнца, способные вызывать изменения океанической циркуляции и влиять на характеристики регионального и глобального климата [4-6].

В ходе предыдущих исследований мы изучили короткопериодические изменения инсоляции, вызванные планетными возмущениями орбиты Земли [7], совместным действием этих возмущений и вариацией солнечной постоянной [8], а также движением системы Земля - Луна вокруг общего центра масс [9]. Было показано, что наиболее заметными короткопериодическими гармониками изменения инсоляции является гармоника $L 0$ с периодом синодического месяца (промежуток времени между одинаковыми фазами Луны - 29d .53 ) и амплитудой $82.54 \pm$ $\pm 0.07 \mathrm{MB} / \mathrm{M}^{2}$, а также гармоника $J 0$, соответствующая синодическому периоду (промежуток времени между одноименными конфигурациями

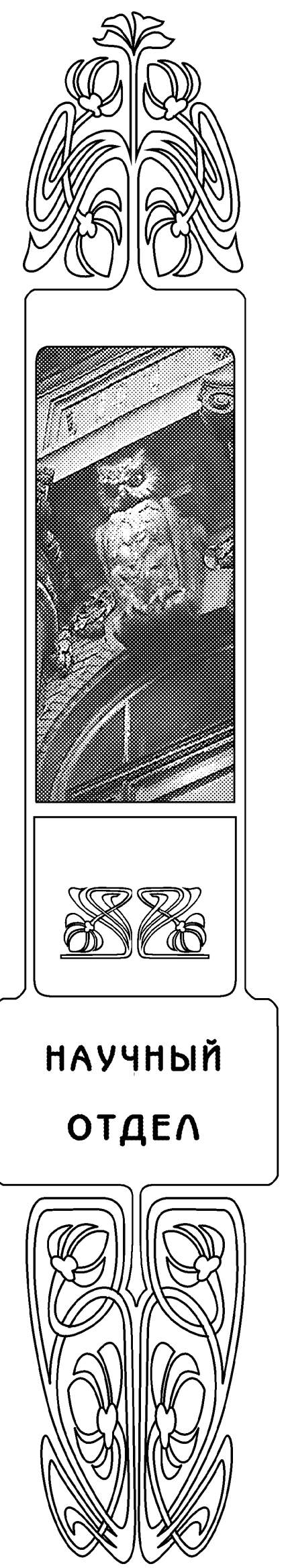


планеты) Юпитера $399^{d} .0$ с амплитудой $44.06 \pm$ $\pm 3.19 \mathrm{MB} / \mathrm{M}^{2}$, и первая гармоника синодического периода Венеры $V 1$ с периодом $291^{\text {d }} .9$ и амплитудой $42.72 \pm 1.12 \mathrm{мBT} / \mathrm{M}^{2}$. Представляет большой интерес попытка обнаружить проявление этих гармоник во временных рядах измерений метеорологических величин.

Целью настоящей работы является поиск возможных периодических составляющих, связанных с изменениями инсоляции и влиянием других космических факторов, в ряде среднесуточных значений приземной температуры воздуха в Саратове, измеренных в период с 1945 по 1999 г.

\section{Исходные данные и их предварительная обработка}

Необходимо отметить, что поставленная нами задача достаточно сложна. Это связано не только с малыми амплитудами гармоник инсоляции, но и с тем, что они действуют на фоне больших изменений температуры, вызванных сменой сезонов года. Кроме того, приземная температура воздуха определяется главным образом процессами циркуляции атмосферы и сильно зависит от состояния облачности. Однако периодический характер гармоник позволяет обнаружить их проявление при анализе достаточно продолжительных временных рядов.

Мы проанализировали ряд среднесуточных значений приземной температуры воздуха, измеренных на метеостанции 34172 Саратов (51⒊ $4^{\prime} \mathrm{N}, 46^{\circ} 02^{\prime} \mathrm{E}$, $\mathrm{H}=156$ м) с 1 октября 1945 г. по 31 августа 1999 г. Информация была получена из ВНИИГМИ - МЦД в рамках проекта European Climate Assessment [10] и в настоящее время доступна по сети Интернет (http:eca.knmi.nl). В нескольких случаях отсутствующие значения были рассчитаны с помощью линей-

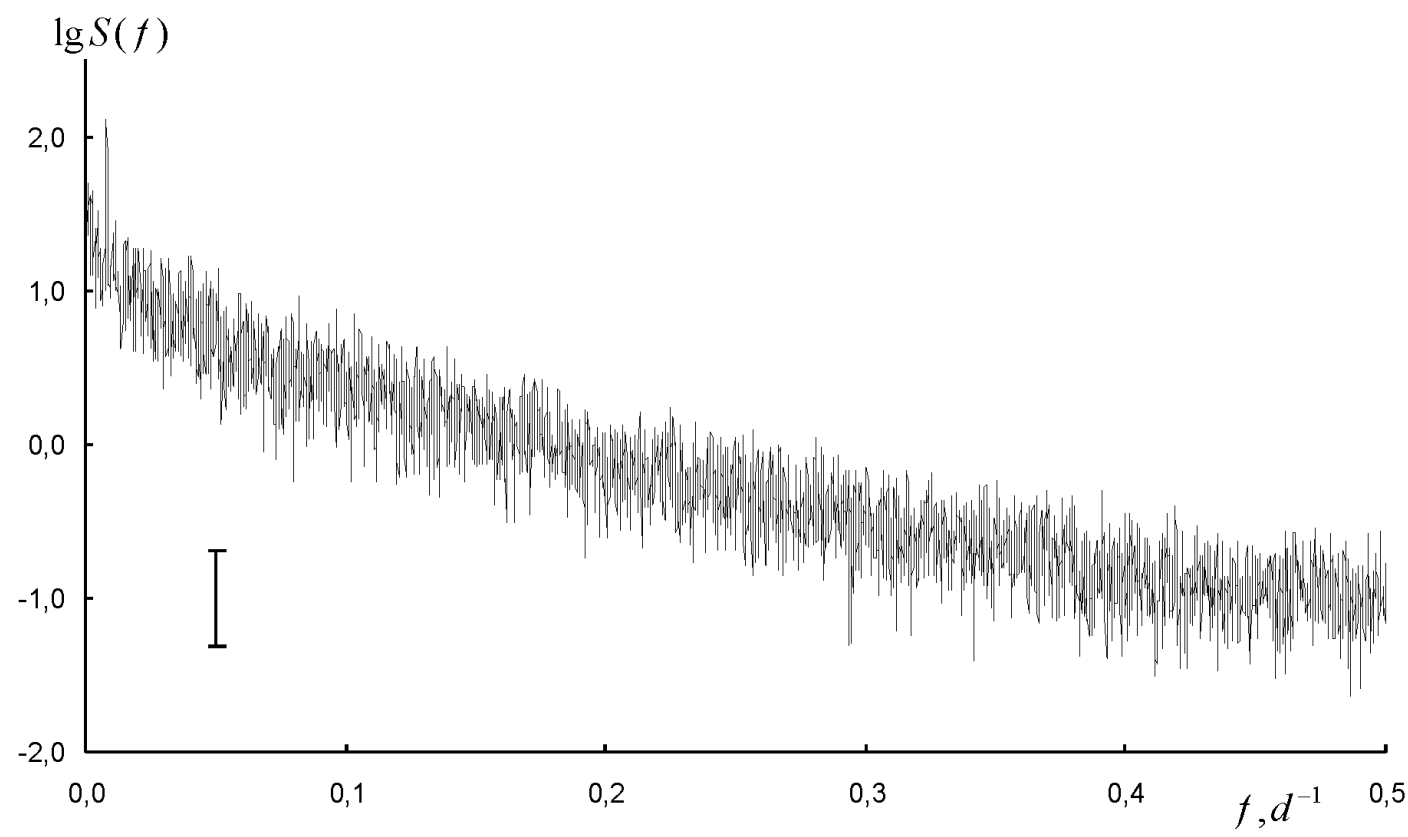

Рис. 1. График логарифма спектра мощности временного ряда изменения приземной температуры воздуха во всем диапазоне частот ной интерполяции данных. Полное число отсчетов ряда составляет 19693.

Для устранения влияния сезонных изменений температуры из ряда был вычтен вклад синусоиды с периодом, равным тропическому году (промежутку времени между двумя последовательными прохождениями Солнца точки весеннего равноденствия - 365. ${ }^{2} 2422$ ). Амплитуда 16.680 K и фаза 2.887 рад. этой синусоиды определялись по способу наименьших квадратов. Аналогичным образом были определены амплитуда 1.028 К и фаза 6.109 рад. первой гармоники тропического года, имеющей период 182. 6211 , которая также была вычтена из ряда среднесуточных значений температуры.

Стандартное отклонение полученного ряда характеризует изменение среднесуточных значений температуры в Саратове, не связанное с сезонными вариациями, и равно 4.93 К.

\section{Анализ спектра мощности временного ряда}

Нами использовался классический вариант цифрового спектрального анализа с расчетом спектра мощности $S(f)$ путем фурье-преобразования автокорреляционной функции [11]. При выбранном максимальном сдвиге автокорреляции в 5000 отсчетов для использованного корреляционного окна Ханна ширина спектрального окна составляет $\Delta f=2.667 \times 10^{-4} \mathrm{~d}^{-1}$. Число степеней свободы спектральной оценки равно 11, а длина $90 \%$ - го логарифмического доверительного интервала - 0.634. График логарифма спектра мощности в диапазоне частот $f$ от нуля до максимальной частоты Найквиста $0.5 \mathrm{~d}^{-1}$, построенный по 5001 расчетной точке, приведен на рис. 1. Вертикальным отрезком на этом рисунке показан логарифмический доверительный интервал. 
В целом характер спектра мощности соответствует чисто случайному процессу, близкому к «красному» шуму, при котором спектральная плотность возрастает с уменьшением частоты. Единственный хорошо заметный пик на частоте $f=0.00821 \mathrm{~d}^{-1}$, соответствующей периоду 121 . d7474, представляет собой вторую гармонику тропического года, не исключавшуюся при предварительной обработке ряда.

Участок спектра мощности в диапазоне частот от нуля до $f=0.01 \mathrm{~d}^{-1}$, содержащий гармоники с периодами $T$, превышающими $100^{\mathrm{d}}$, приведен на рис. 2. Вертикальным отрезком на этом рисунке показан логарифмический доверительный интервал, а горизонтальным - ширина спектрального окна, характеризующая разрешающую способность по частоте. Единственный статистически значимый пик соответствует второй гармонике тропического года. Анализ этого участка спектра свидетельствует об отсутствии пиков на частотах, соответствующих 11-летнему циклу солнечной активности, синоди- ческому периоду Юпитера $J 0$ и первой гармонике синодического периода Венеры V1. Если для обнаружения проявления солнечного цикла длина нашего ряда может считаться недостаточной, то отсутствие гармоник планетных возмущений свидетельствует либо о том, что их влияние слишком мало, либо оно в значительной степени подавляется шумовыми флуктуациями.

На рис. 2 отсутствуют также пики, соответствующие квазидвухлетнему циклу (QBO, $T=$ $=2.40$ года) и чандлеровскому периоду движения полюса Земли ( $T=1.19$ года). Квазидвухлетний цикл был обнаружен по изменениям скорости ветра в экваториальной стратосфере и часто проявляется в различных геофизических явлениях [3]. $\mathrm{C}$ чандлеровским периодом связан так называемый полюсный прилив, влияющий также на изменение приземного атмосферного давления [12]. Данный результат свидетельствует о слабости влияния этих факторов на температуру нижних слоев атмосферы.

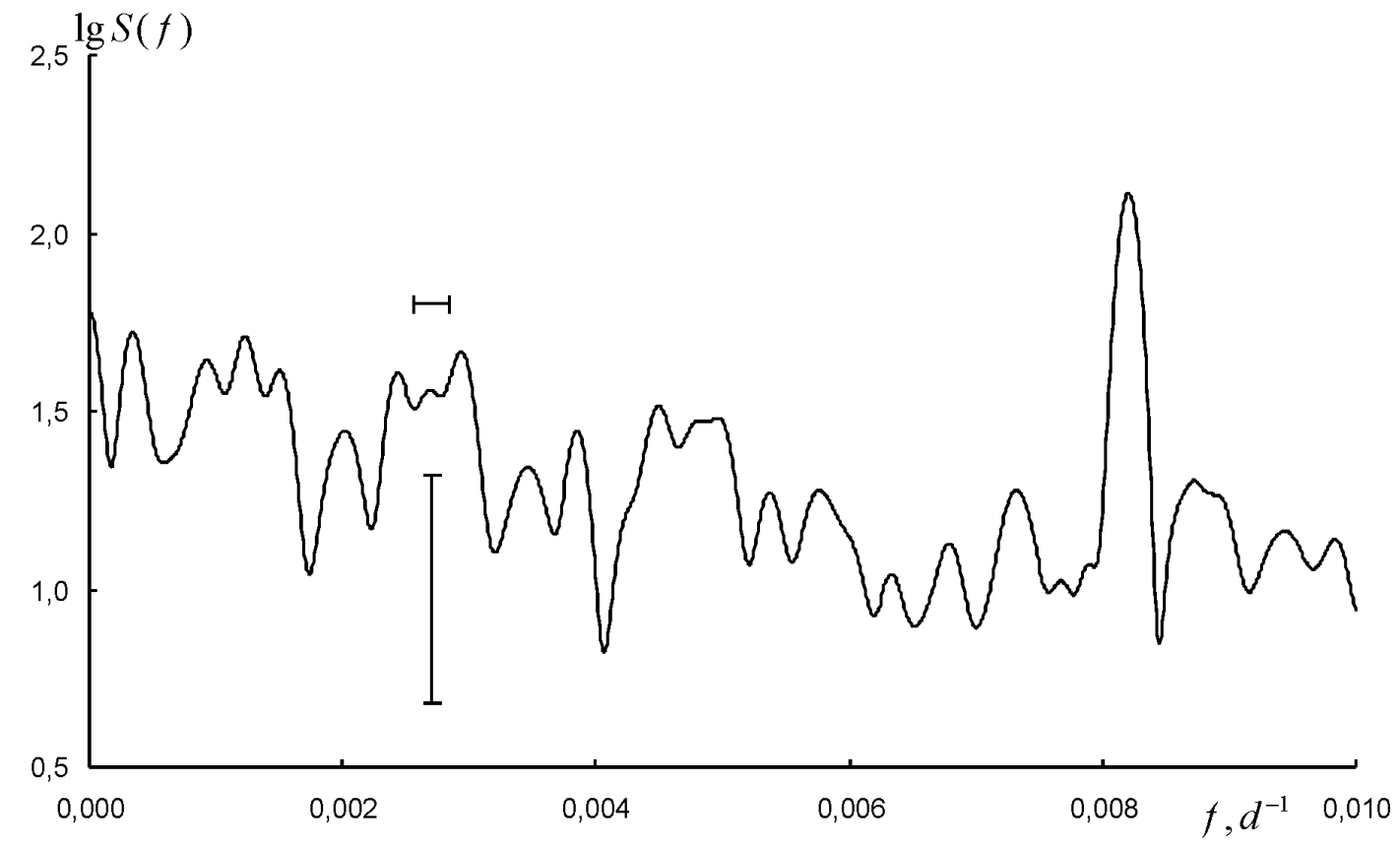

Рис. 2. График логарифма спектра мощности в диапазоне гармоник с периодами, превышающими $100^{\mathrm{d}}$. Единственный значимый пик соответствует второй гармонике тропического года

Область спектра в диапазоне частот $f$ от $0.03 \mathrm{~d}^{-1}$ до $0.04 \mathrm{~d}^{-1}$, соответствующих периодам около одного месяца, приведена на рис. 3 . Совмещая середину доверительного интервала с основанием пиков в спектре мощности можно убедиться, что ряд наблюдаемых гармоник являются статистически значимыми. Если принять, что погрешность оценки частоты гармоники $f_{m}$, соответствующей максимуму пика, равна ширине спектрального окна $\Delta f$, то погрешность определения соответствующего периода $T=1 / f_{m}$ будет равна $\Delta T=T^{2} \Delta f$. Считая, что найденные периоды гармоник должны отличаться от точных значений не более чем на величину $\Delta T$, мы можем отождествить в данной области спектра три периодических составляющих: гармонику синодического месяца $L 0\left(T=29^{\mathrm{d}} .53\right)$, приливную гармонику $M_{m}$ $\left(T=27^{\mathrm{d}} .55\right)$ и гармонику, связанную с солнечной активностью, $T_{\odot}\left(T=27^{\mathrm{d}} .0\right)$. Обозначения этих гармоник показаны у вершин соответствующих пиков на рис. 3 .

Данные об отождествленных гармониках приведены в таблице. В первом столбце дано обозначение гармоники, во втором - частота $f_{m}$, соответствующая максимуму спектрального пика, в третьем - найденное значение периода $T$ 


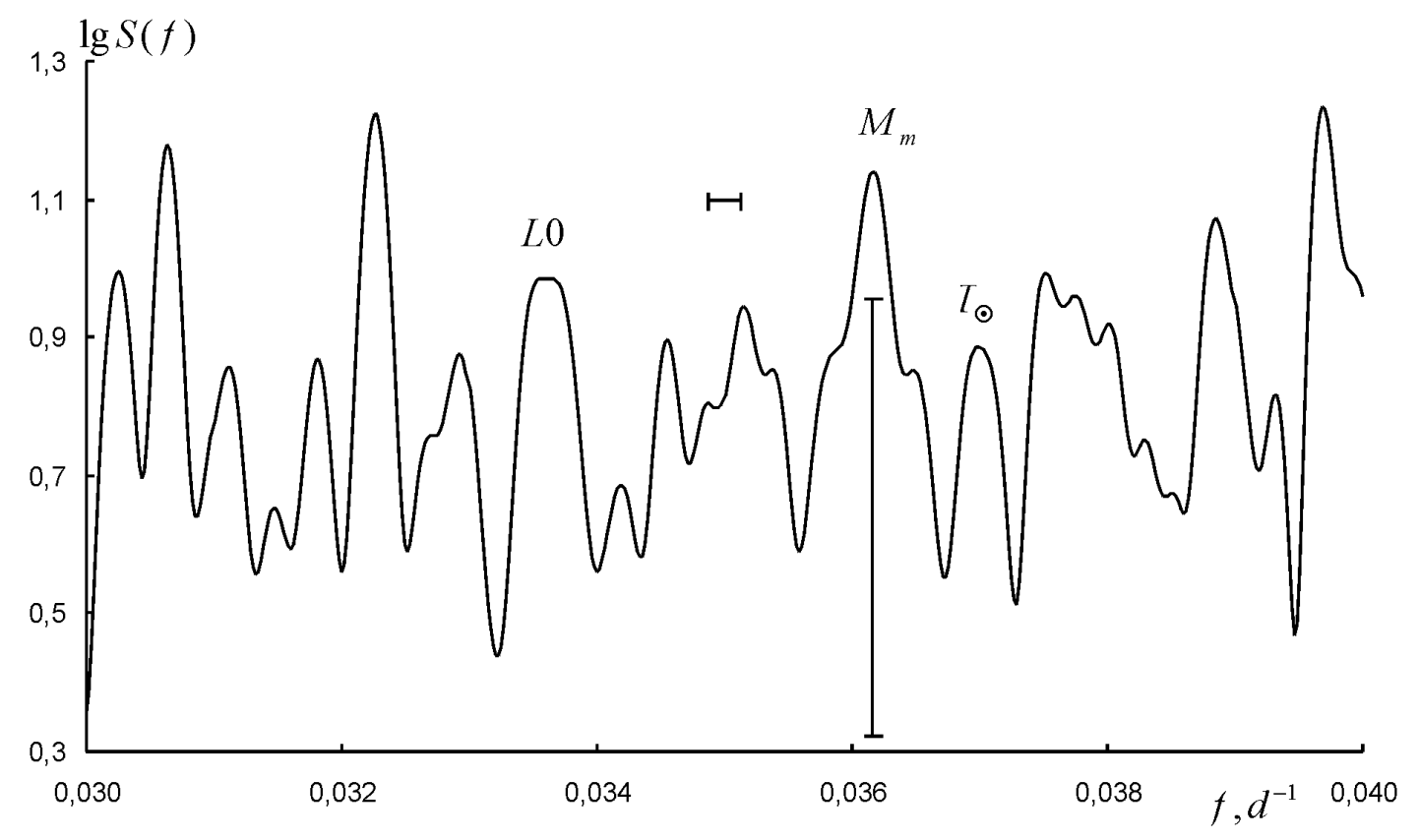

Рис. 3. График логарифма спектра мощности в диапазоне гармоник с периодами около месяца. Отмечены пики, соответствующие гармонике синодического месяца $L 0$, приливной гармонике $M_{m}$, а также гармонике $T_{\odot}$, совпадающей со средним периодом обращения активных областей Солнца

с его стандартным уклонением $\Delta T$, а в четвертом - амплитуда, измеренная в кельвинах. В качестве оценки амплитуды гармоники нами бралась величина

$$
A\left(f_{m}\right)=\sigma \sqrt{2 \Delta f\left(S\left(f_{m}\right)-S_{0}\right)},
$$

где $\sigma$ - среднеквадратичное отклонение временного ряда, равное $4.93 \mathrm{~K} ; \Delta f$ - ширина спектрального окна; $S\left(f_{m}\right)$ - значение спектральной плотности в максимуме пика; а $S_{0}$ - величина спектральной плотности у основания пика, оцениваемая как полусумма значений $S(f)$, измеренных слева и справа от пика. Известно, что при цифровом спектральном анализе частоты гармоник определяются гораздо точнее, чем их амплитуды. С учетом величины доверительного интервала спектральной оценки можно утверждать, что с вероятностью 90\% амплитуды гармоник должны отличаться от найденных значений не более чем в два раза.

Характеристики гармоник, найденных в спектре мощности временного ряда изменения приземной температуры воздуха

\begin{tabular}{|c|c|c|c|}
\hline Гармоника & $f_{m}, \mathrm{~d}^{-1}$ & $T, \mathrm{~d}$ & $A\left(f_{m}\right), \mathrm{K}$ \\
\hline$L 0$ & 0.03364 & $29.73 \pm 0.24$ & 0.29 \\
$M_{m}$ & 0.03616 & $27.65 \pm 0.20$ & 0.36 \\
$T_{\odot}$ & 0.03700 & $27.03 \pm 0.19$ & 0.24 \\
$M_{f}$ & 0.07332 & $13.64 \pm 0.05$ & 0.16 \\
\hline
\end{tabular}

Область спектра в диапазоне частот $f$ от $0.07 \mathrm{~d}^{-1}$ до $0.08 \mathrm{~d}^{-1}$, соответствующих периодам около полумесяца приведена на рис. 4. Данная область содержит пик с частотой $0.07332 \mathrm{~d}^{-1}$, соответствующий приливной гармонике $M_{f}(T=$ $\left.=13^{\mathrm{d}} .66\right)$. Характеристики этой гармоники также приведены в таблице.

\section{Обсуждение результатов}

Несмотря на то что не все спектральные пики обнаруженных гармоник заметно выходят за границу принятого доверительного интервала, хоро- шее совпадение найденных периодов с точными значениями служит дополнительным аргументом в пользу их достоверности.

Очевидным объяснением присутствия во флуктуациях приземной температуры воздуха гармоники $L 0$ с периодом синодического месяца является изменение инсоляции при движении системы Земля - Луна вокруг общего центра масс с амплитудой $82.54 \pm 0.07 \mathrm{MBT} / \mathrm{M}^{2}$ [9]. Существует еще один космический фактор, способный повлиять на температуру атмосферы, который действует синхронно с данным изменением инсоляции. Речь идет об излучении Луны, зависящем от ее фазы и 


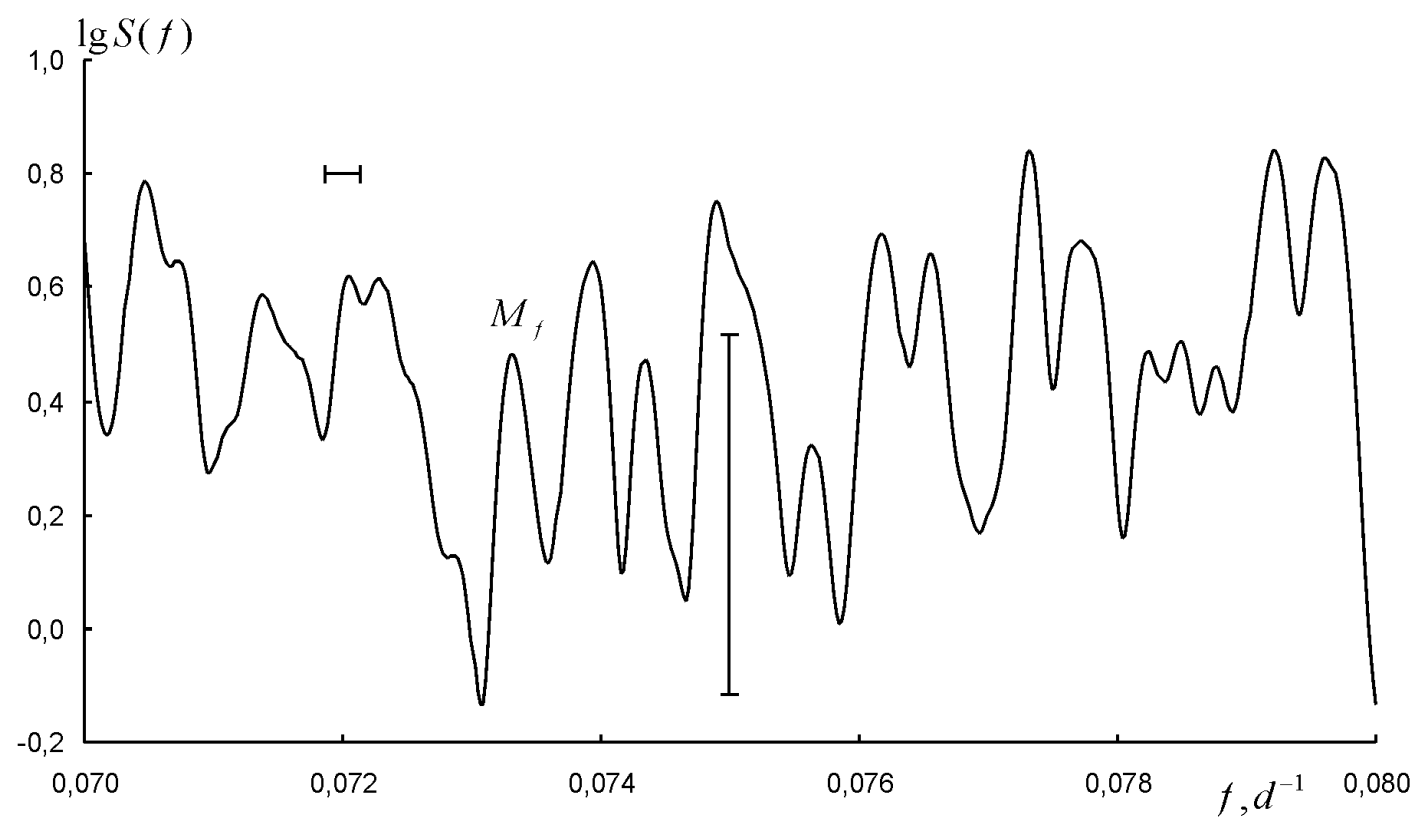

Рис. 4. График логарифма спектра мощности в диапазоне гармоник с периодами около полумесяца. Отмечен пик, соответствующий приливной гармонике $M_{f}$

достигающего максимума в момент полнолуния. Однако влияние этого фактора достаточно мало. В видимом диапазоне спектра (фотометрическая полоса $V$ ) звездные величины полной Луны и Солнца равны соответственно $V_{L}=-12.73$ и $V_{\odot}=-26.74[13]$, что дает отношение потоков излучения от этих небесных тел $0.25 \times 10^{-5}$. Следует однако учесть, что альбедо Луны мало $(\approx 0.07)$ и основная часть освещающего ее солнечного излучения поглощается поверхностью нашего спутника. Нагретая поверхность Луны излучает в ИК-диапазоне спектра. Поэтому отношение болометрических потоков должно быть больше. По данным радиометрических измерений [14], для среднего полнолуния оно равно $0.96 \times 10^{-5}$, что с учетом величины солнечной постоянной $1366.22 \mathrm{BT} / \mathrm{M}^{2}$ дает поток от Луны $13 \mathrm{MBT} / \mathrm{M}^{2}$. В фазе новолуния поток от Луны очень мал. Поэтому в качестве верхнего предела амплитуды изменения потока ее излучения можно принять значение $6.5 \mathrm{mBT} / \mathrm{M}^{2}$, что более чем на порядок меньше амплитуды изменения инсоляции при движении системы Земля - Луна вокруг общего центра масс.

Присутствие в спектре мощности нашего временного ряда гармоник лунно-солнечных приливов $M_{m}$ и $M_{f}$ не является неожиданным. Атмосферные приливы вызывают изменение давления, что должно сопровождаться и изменением температуры. Теория этих приливов достаточно хорошо разработана. Однако в известной монографии [15] и более поздних исследованиях [16] рассматривались только наиболее мощные гармоники гравитационных и термических приливов с суточным и меньшими периодами. Было бы ин- тересно провести аналогичные расчеты амплитуд приземных температур воздуха, связанных с месячной и полумесячной приливными гармониками для сравнения с нашими результатами.

Вызванная солнечной активностью гармоника $T_{\odot}$ имеет период $T=27^{\mathrm{d}} .0$, совпадающий со средним периодом обращения активных областей Солнца [17]. Дать однозначное объяснение появлению этой гармоники в ряде приземных температур воздуха достаточно трудно. С одной стороны, здесь, возможно, проявляет себя блокировка излучения фотосферы, вызванная наличием групп пятен. С другой - в районе этих активных областей происходят хромосферные вспышки, сопровождающиеся резким усилением жесткого УФ-излучения, а иногда и рентгеновского. Излучение этих диапазонов спектра поглощается верхними слоями земной атмосферы, вызывая их заметный нагрев. Однако до настоящего времени не известен сколь-либо эффективный механизм передачи этой энергии к поверхности Земли. Оба указанных фактора солнечной активности могут действовать и совместно.

\section{Заключение}

Проведенный анализ спектра мощности временного ряда среднесуточных значений приземной температуры воздуха в Саратове в период с 1945 по 1999 г. позволил достаточно надежно обнаружить присутствие в изменениях температуры периодических составляющих. В спектре ряда найдены две приливные гармоники $M_{m}$ и $M_{f}$, гармоника с периодом синодического месяца $L O$, связанная с изменением инсоляции при движении системы 
Земля - Луна вокруг общего центра масс, а также гармоника $T_{\odot}$, соответствующая среднему периоду обращения активных областей Солнца.

Проявлений 11-летнего цикла солнечной активности и периодических изменений инсоляции, вызванных планетными возмущениями орбиты Земли, нами не обнаружено. В ходе солнечного цикла, наряду с большими вариациями потоков жесткого УФ-излучения и рентгеновского, наблюдается также изменение инсоляции с амплитудой около $1 \mathrm{BT} / \mathrm{M}^{2}$. Отсутствие проявлений этого цикла можно было бы объяснить сравнительно малой длиной нашего ряда. Однако анализ более продолжительных рядов среднегодовых температур различных пунктов также не дает однозначных свидетельств в пользу связи их изменений с солнечной активностью [17].

Две главные гармоники изменения инсоляции $J 0$ и $V 1$, вызванные планетными возмущениями, имеют периоды около года и вполне могли бы проявить себя. Отсутствие в спектре мощности соответствующих пиков свидетельствует либо о том, что их влияние слишком мало, либо оно в значительной степени подавляется шумовыми флуктуациями. То же самое можно сказать в отношении гармоник, соответствующих квазидвухлетнему циклу и чандлеровскому периоду движения полюса Земли. Хотя эти циклы часто проявляются в различных геофизических явлениях, отсутствие соответствующих пиков в спектре свидетельствует о слабости их влияния на температуру нижних слоев атмосферы.

\section{Библиографический список}

1. Авдюшин С.И., Данилов А.Д. Солнце, погода и климат: сегодняшний взгляд на проблему. (Обзор) // Геомагнетизм и аэрономия. 2000. Т. 40, № 5. С. 3-14.

2. Монин А.С., Шишков Ю.А. Климат как проблема физики // Успехи физ. наук. 2000. Т. 170, № 4. С. 419-445.

3. Сидоренков Н.С. Атмосферные процессы и вращение Земли. СПб.: Гидрометеоиздат, 2002. 200 с.
4. Авсюк Ю.Н. Приливные силы и природные процессы. М.: Объединенный ин-т физики Земли им. О.Ю. Шмидта, $1996.188 \mathrm{c}$.

5. Wunsch C. Moon, tides and climate // Nature. 2000. Vol. 405, P. 743-744.

6. Treloar N.C. Luni - solar tidal influences on climate variability // Intern. J. of Climatology. 2002. Vol. 22, № 12. P. $1527-1542$.

7. Богданов М.Б., Сурков А.Н. Короткопериодные изменения инсоляции, вызванные планетными возмущениями орбиты Земли // Метеорология и гидрология. 2006. № 1. C. $48-54$.

8. Богданов М.Б., Катрущенко А.В., Сурков А.Н. Изменения инсоляции, вызванные планетными возмущениями орбиты Земли и вариацией солнечной постоянной // Изв. Сарат. ун-та. Сер. Науки о Земле. 2006. Т. 6, вып. 1. C. 3-9.

9. Богданов М.Б., Катрущенко А.В. Изменения инсоляции, вызванные влиянием Луны // Изв. Сарат. ун-та. Сер. Науки о Земле. 2008. Т. 8, вып. 1. С. 3-6.

10. Klein Tank A.M.G., Wijngaard J.B., Konnen G.P. et al. Daily dataset of 20th-century surface air temperature and precipitation series for the European Climate Assessment // Intern. J. of Climatology. 2002. Vol. 22. P. 1441-1453.

11. Дженкинс Г., Ваттс Д. Спектральный анализ и его приложения. М.: Мир, 1972. 603 с.

12. Максимов И.В. Геофизические силы и воды океана. Л.: Гидрометеоиздат, $1970.447 \mathrm{c.}$

13. Аллен К.У. Астрофизические величины. М.: Мир, 1977. $446 \mathrm{c}$.

14. Pettit E. Lunar radiation as related to phase // Astrophys. J. 1935. Vol. 81. P. 17-36.

15. Чепмен $C$., Линдзен Р. Атмосферные приливы: термические и гравитационные. М.: Мир, 1972. 296 с.

16. Zurek R.W. The form of Newtonian cooling in atmospheric tidal theory // Pure and Applied Geophys. 1985. Vol. 123. P. 921-929.

17. Герман Дж.Р., Голдберг Р.А. Солнце, погода и климат. Л.: Гидрометеоиздат, $1981.319 \mathrm{c.}$

\section{УДК 502.3}

\section{СПОСОБНОСТЬ АТМОСФЕРЫ РАЗЛИЧНЫХ РАЙОНОВ САРАТОВСКОЙ ОБЛАСТИ К САМООЧИЩЕНИЮ}

\section{С.Н. Лапина, Е.А. Полянская, Л.М. Фетисова, Н.А. Фетисова}

Саратовский государственный университет, кафедра метеорологии и климатологии E-mail: kafmeteo@sgu.ru

В статье приводятся результаты исследования способности атмосферы к самоочищению в Саратове и Саратовской области. Проанализирована динамика коэффициента самоочищения атмосферы в различных районах Саратовской области в теплый и холодный сезоны за многолетний период и 2000-2004 гг. Формула расчета коэффициента самоочищения атмосферы адап- тирована к региональным климатическим условиям. Построены карты-схемы районирования территории Саратова по коэффициенту самоочищения атмосферы. Исследование метеорологического потенциала самоочищения атмосферы имеет практическое значение при определении оптимального распределения техногенных нагрузок на окружающую среду. 